\title{
A CLASS OF INEQUALITIES RELATING DEGREES OF ADJACENT NODES TO THE AVERAGE DEGREE IN EDGE-WEIGHTED UNIFORM HYPERGRAPHS
}

\author{
P. D. JOHNSON JR. AND R. N. MOHAPATRA
}

Received 26 August 2004 and in revised form 28 September 2005

In 1986, Johnson and Perry proved a class of inequalities for uniform hypergraphs which included the following: for any such hypergraph, the geometric mean over the hyperedges of the geometric means of the degrees of the nodes on the hyperedge is no less than the average degree in the hypergraph, with equality only if the hypergraph is regular. Here, we prove a wider class of inequalities in a wider context, that of edge-weighted uniform hypergraphs.

\section{Definitions and preliminary observations}

A $k$-uniform hypergraph is a pair $(V, E)=G$ in which $V$, the set of vertices or nodes of $G$, is a set, and $E$, the set of hyperedges or edges of $G$, is a multiset of multisets of elements of $V$, with each $e \in E$ having multiset cardinality $k$. When $k=2$, such an object is called a graph, or multigraph, and, in this case, when $E$ and every element of $E$ are sets, then $G$ is called a simple graph.

Throughout this paper, $G=(V, E)$ will be a finite $k$-uniform hypergraph of order $n=$ $|V| ;|E|$ will be denoted by $m$. We require that every vertex appears on some edge. A weighting of the edges of $G$ is a function $w t: E \rightarrow I$, where $I$ is a real interval, usually $[0, \infty)$. For such a weighting, for any submultiset $E^{\prime}$ of $E, w t\left(E^{\prime}\right)=\sum_{e \in E^{\prime}} w t(e)$. We will refer to the pair $(G, w t)$ as an edge-weighted hypernetwork, or, more simply, as a weighted network. Given $(G, w t)$ and $v \in V$, the degree of $v$ in $(G, w t)$ is the sum of the weights of the edges to which $v$ belongs, where each weight is counted according to the multiplicity of $v$ on the edge. More formally, for $v \in V$ and $e \in E$, let $\delta(v, e)$ be the number of times that $v$ occurs in the multiset $e$. Then the degree of $v$ in $(G, w t)$ is

$$
d(v)=\sum_{e \in E} \delta(v, e) w t(e)
$$

The average degree in $(G, w t)$ is $\bar{d}=(1 / n) \sum_{v \in V} d(v)$. When $d$ is a constant function, $(G, w t)$ is regular, with degree $d=\bar{d}$. When $w t \equiv 1$, the degree in the weighted network is just the ordinary degree in $G$, denoted by $d_{G}$. 
The $k$-uniformity of the hypergraph $G$ implies a simple relation among $\bar{d}, n$, and $w t(E)$ :

$$
\begin{aligned}
\bar{d} & =\frac{1}{n} \sum_{v \in V} d(v)=\frac{1}{n} \sum_{v \in V} \sum_{e \in E} \delta(v, e) w t(e) \\
& =\frac{1}{n} \sum_{e \in E}\left(\sum_{v \in V} \delta(v, e)\right) w t(e)=\frac{k}{n} \sum_{e \in E} w t(e) \\
& =\frac{k}{n} w t(E) .
\end{aligned}
$$

For $e \in E$, let $u_{1}(e), \ldots, u_{k}(e)$ be the $k$ vertices on $e$ (repeated according to their multiplicities), in no particular order. Suppose that $F$ is a symmetric function of $k$ real variables. The inequality form announced in the title is $*(G, w t, F)$ :

$$
w t(E)^{-1} \sum_{e \in E} w t(e) F\left(d\left(u_{1}(e)\right), \ldots, d\left(u_{k}(e)\right)\right) \geq F(\bar{d}, \ldots, \bar{d})
$$

(Arrangements will be made so that all the $k$-tuples $\left(d\left(u_{1}(e)\right), \ldots, d\left(u_{k}(e)\right)\right)$ and $(\bar{d}, \ldots$, $\bar{d})$ are in the domain of $F$.) Notice that $*(G, w t, F)$ holds with equality whenever $(G, w t)$ is regular. We will be especially interested in families of weighted networks $(G, w t)$ for which $*(G, w t, F)$ holds, for some particular $F$, with equality only when $(G, w t)$ is regular.

Why the interest in inequalities of this form? One reason is that there are some quite memorable and useful inequalities of this form. For instance, in [2], it is shown that $*(G, w t, F)$ holds strictly for all nonregular $k$-uniform $G, w t \equiv 1$, and $F\left(x_{1}, \ldots, x_{k}\right)=$ $\sum_{i=1}^{k} \log \left(x_{i}\right)$; manipulating this result, we obtain, with $d=d_{G}$,

$$
\left[\Pi_{e \in E}\left[\Pi_{j=1}^{k} d\left(u_{j}(e)\right)\right]^{1 / k}\right]^{1 /|E|} \geq \bar{d}
$$

with equality only when $G=(G, 1)$ is regular. This says that the geometric mean, over the edges, of the geometric means of the degrees of the vertices on each edge is no less than the arithmetic average degree (with equality only when $G$ is regular), which seems, at first glance, to reverse the conventional wisdom about geometric means and arithmetic means. A moment's thought shows that (1.4) is not very surprising, since vertices contribute as many factors to the product on the left-hand side of (1.4) as their degree, which gives rich vertices of high degree an undemocratic advantage in hoisting the result. Still, surprising or not, it is a pretty and inobvious inequality. Corollary 2.3 is a generalization of it.

Obviously, there is a rich trove of problems under the general question of when-for which $G, w t$, and $F$ - the inequality $*(G, w t, F)$ (i.e., (1.3)) holds, and the main result here is just one small answer, a characterization of functions $F$ of a very particular form 
such that $*(G, w t, F)$ holds for all $G$ and $w t$. Before getting to that result, however, we make two easy remarks that bear on the general question.

(1) In defining $k$-uniform hypergraphs, we allowed $E$ to be a multiset, that is, hyperedges can be repeated. Not only is this customary, but it also seemed unavoidable, for full generality, in the main result in [2]. It is a happy consequence of the generalization to weighted networks that we can simplify to the cases where $E$ is merely a set. Suppose $E$ is a multiset, and $*(G, w t, F)$ holds for some $w t, F$. Let $\hat{G}$ be obtained by collapsing each multiple (repeated) hyperedge to a single hyperedge, and let $\widehat{w t}$ be the weighting of the hyperedges of $\hat{G}$ in which each hyperedge is weighted with the sum of the $w t$ values on its copies in $E$. Then $\widehat{G}$ has the same vertices as $G$, with the same degrees, and the fact that multiplication distributes over addition shows that $*(\widehat{G}, \widehat{w t}, F)$ holds. The same observation shows that going the other way, splitting a weighted edge into copies and distributing its weight over the copies, preserves the inequality.

(2) Suppose that 0 is allowed as an edge weight, and suppose that $*(G, w t, F)$ holds, for some $F$, and for all $w t$, or perhaps for all $w t$ in some reasonable class, such as the class of weightings with respect to which every vertex has positive degree. (If, e.g., $F\left(x_{1}, \ldots, x_{k}\right)$ is defined only for $x_{1}, \ldots, x_{k}>0$, and if 0 is allowed as an edge weight, then $*(G, w t, F)$ would make sense only for weightings in that class.) Then we can conclude, if the class of weightings $w t$ for which $*(G, w t, F)$ holds permits, that $*(H, w t, F)$ holds for all suitable $w t$, and for all spanning subhypergraphs $H$ of $G$. A spanning subhypergraph of $G$ is one obtained by deleting edges of $G$, but so that each vertex of $G$ still lies on at least one of the edges remaining. Given such a spanning subhypergraph $H$, and a weighting $w t$ of the edges of $H$, extend the weighting to a weighting of $G$ simply by weighting each edge of $G$ which is not in $H$ with zero. Then, $*(G, w t, F)$ clearly implies $*(H, w t, F)$, since the degrees of the vertices with respect to $w t$ are the same, in $G$ and $H$, and the terms on the left-hand side of the inequality corresponding to edges in $G$, but not $H$, are zero.

Observations (1) and (2) are trivial, but useful. For instance, to prove that $*(G, w t, F)$ holds, for some particular $F$, for all $w t$ (or, all $w t$ such that every degree is positive), and for all loopless multigraphs ( $k=2$ and loops and isolates are not allowed) $G$, it suffices to verify the inequality for all simple complete graphs. To prove that the inequality holds for all $w t$ and all multigraphs $G$, it suffices to show that it holds for all $w t$ and $G$ ranging over the simple complete graphs with a loop added at each vertex.

\section{Results and proofs}

A real-valued function $f$ is convex on an interval $I$ if and only if

$$
f(t x+(1-t) y) \leq t f(x)+(1-t) f(y) \quad \forall x, y \in I, t \in[0,1]
$$

If $t \in(0,1)$ and $x \neq y$ imply strict inequality, then $f$ is said to be strictly convex. There is an easy test for convexity: if $f$ is continuous on $I$ and $f^{\prime \prime}$ is defined and nonnegative on the interior of $I$, then $f$ is convex on $I$. If $f^{\prime \prime}>0$ on the interior of $I$, and $f$ is continuous on $I$, then $f$ is strictly convex on $I$. 
If $f$ is convex on $I, x_{1}, \ldots, x_{n} \in I, \lambda_{1}, \ldots, \lambda_{n}>0$, and $\sum_{i=1}^{n} \lambda_{i}=1$, then

$$
f\left(\sum_{i=1}^{n} \lambda_{i} x_{i}\right) \leq \sum_{i=1}^{n} \lambda_{i} f\left(x_{i}\right) .
$$

If $f$ is strictly convex on $I$, then equality holds in the inequality just above if and only if $x_{1}=\cdots=x_{k}$. This inequality is known as Jensen's inequality (see [1], pages 70-74). We will be using it first with $\lambda_{1}=\cdots=\lambda_{n}=1 / n$.

The following lemma is actually a generalization of most of the theorem to follow. We have relegated it to lemma status because it is quite unmemorable.

Lemma 2.1. Suppose that $A=\left[a_{i j}\right]$ is an $n \times m$ matrix of real numbers with constant column sum $c$; that $\varphi$ is a real-valued function on a real interval I such that $f$, defined by $f(x)=x \varphi(x)$, is convex on $I$; and that $\left(x_{1}, \ldots, x_{m}\right)$ is an $m$-tuple of real numbers such that $s=\sum_{j=1}^{m} x_{j}>0$ and $d_{i}=\sum_{j=1}^{m} a_{i j} x_{j} \in I$ for each $i=1, \ldots, n$. Let $\bar{d}=(1 / n) \sum_{i=1}^{n} d_{i}$. Then $s^{-1} \sum_{j=1}^{m} \sum_{i=1}^{n} \varphi\left(d_{i}\right) a_{i j} x_{j} \geq c \varphi(\bar{d})$, and if $f$ is strictly convex, then equality holds only if $d_{1}=\cdots=d_{n}$.

Proof. First note that $\bar{d}=(1 / n) \sum_{i=1}^{n} \sum_{j=1}^{m} a_{i j} x_{j}=(1 / n) \sum_{j=1}^{m}\left(\sum_{i=1}^{n} a_{i j}\right) x_{j}=(c / n) s$. With this in mind, we have

$$
\begin{aligned}
s^{-1} \sum_{j=1}^{m} \sum_{i=1}^{n} \varphi\left(d_{i}\right) a_{i j} x_{j} & =s^{-1} \sum_{i=1}^{n}\left(\sum_{j=1}^{m} a_{i j} x_{j}\right) \varphi\left(d_{i}\right)=s^{-1} \sum_{i=1}^{n} d_{i} \varphi\left(d_{i}\right) \\
& =s^{-1} \sum_{i=1}^{n} f\left(d_{i}\right) \geq s^{-1} n f(\bar{d})=s^{-1} n \bar{d} \varphi(\bar{d}) \\
& =s^{-1} n \frac{c}{n} s \varphi(\bar{d})=c \varphi(\bar{d}),
\end{aligned}
$$

by the presumed convexity of $f$, and if $f$ is strictly convex, then equality forces the $d_{i}$ to be equal.

THEOREM 2.2. Suppose that $\varphi$ is a real-valued function on a real interval I such that $f(x)=$ $x \varphi(x)$ is convex on $I$. For each integer $k \geq 1$, and each $k$-uniform hypergraph $G=(V, E)$, and each weighting wt of the edges of $G$ such that $w t(E)>0$, and the weighted degrees of vertices are all in $I$, the inequality $*(G, w t, F)$ holds, with $F$ defined by $F\left(x_{1}, \ldots, x_{k}\right)=\sum_{i=1}^{k} \varphi\left(x_{i}\right)$. Further, if $f$ is strictly convex, then equality implies that the weighted network $(G, w t)$ is regular.

If $I \subseteq[0, \infty), \varphi$ is bounded on each compact subinterval of I and $*\left(C_{4}, w t, F\right)$ holds for all admissible edge weightings of $C_{4}$, the simple cycle of length $4(k=2)$, then $f(x)=x \varphi(x)$ is convex. Further, if equality implies that $\left(C_{4}, w t\right)$ is regular, then $f$ is strictly convex.

Proof. The main claim of the theorem follows from the lemma, taking $A$ to be the vertexedge incidence matrix of $G$. The rows of this graph are indexed by the vertices, the columns by the edges, and the entry at position $(v, e)$ is $\delta(v, e)$, the number of times $v$ appears in the multiset $e$. The assumption of $k$-uniformity says that the column sums are 
all equal to $k$. For a weighting $w t$ satisfying the hypothesis of the theorem, let $(w t(e))_{e \in E}$ play the role of $\left(x_{1}, \ldots, x_{m}\right)$ in the lemma. Then $s=w t(E)$, and for $v \in V$,

$$
d_{v}=\sum_{e \in E} \delta(v, e) w t(e)=d(v)
$$

Further, $\sum_{e \in E} \sum_{v \in V} \varphi(d(v)) \delta(v, e) w t(e)=\sum_{e \in E} w t(e) F\left(u_{1}(e), \ldots, u_{k}(e)\right)$. Thus, the inequality concluded in the lemma is

$$
w t(E)^{-1} \sum_{e \in E} w t(e) F\left(u_{1}(e), \ldots, u_{k}(e)\right) \geq k \varphi(\bar{d})=F(\bar{d}, \ldots, \bar{d})
$$

and the conclusion in case of strict convexity follows, as well.

Now suppose that $I \subseteq[0, \infty), \varphi$ is "locally bounded," as hypothesized, and $*\left(C_{4}, w t, F\right)$ holds for all weightings $w t$ such that $w t(E)>0$ and the weighted degrees of the vertices of $C_{4}$ are all in $I$. Then, $f$ is locally bounded. It is an elementary exercise to verify that, in this case, to show that $f$ is convex, it suffices to show that $f$ is midpoint convex, that is, that $f((x+y) / 2) \leq(1 / 2)(f(x)+f(y))$ for all $x, y \in I$. Suppose that $x, y \in I$; we may as well suppose that $x \neq y$. Weight the edges of $C_{4}$ as depicted:

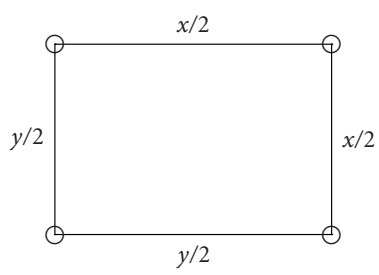

Then, $I \subseteq[0, \infty)$ and $x \neq y$ imply that $w t(E)=x+y>0$, and the degrees of the vertices of $C_{4}, x, y$, and $(x+y) / 2$ are all in $I$. The inequality $*\left(C_{4}, w t, F\right)$ asserts that $(1 /(x+$ $y))[2(x / 2)(\varphi(x)+\varphi((x+y) / 2))+2(y / 2)(\varphi(y)+\varphi((x+y) / 2))] \geq 2 \varphi((x+y) / 2)$, which rearranges to $f(x)+f(y) \geq 2 f((x+y) / 2)$, which verifies that $f$ is midpoint convex, and therefore convex.

If $f$ is convex but not strictly convex, then $f(x)=a x+b$ for all $x$ in some subinterval of $I$, for some constants $a$ and $b$. Choosing $x$ and $y, x \neq y$, from that subinterval, and weighting the edges of $C_{4}$ as above, we have equality in $*\left(C_{4}, w t, F\right)$, yet $\left(C_{4}, w t\right)$ is not regular.

Corollary 2.3. Suppose that $k \geq 1, G=(V, E)$ is a $k$-uniform hypergraph, and wt is a weighting of the edges of $G$ with real weights so that the degree of each vertex of $G$ in $(G, w t)$ is positive. Then

$$
\left[\Pi_{e \in E}\left(\Pi_{j=1}^{k} d\left(u_{j}(e)\right)\right)^{w t(e) / k}\right]^{1 / w t(E)} \geq \bar{d}
$$

with equality only if $(G, w t)$ is regular.

Proof. Apply the theorem with $\varphi(x)=\log x$. Note that $\bar{d}>0$ implies that $w t(E)>0$. 
Corollary 2.4. With $k, G$, and wt as in Corollary 2.3, suppose that $p>0$. Then

$$
\left[\frac{1}{w t(E)} \sum_{e \in E} w t(e)\left(\frac{1}{k} \sum_{j=1}^{k} d\left(u_{j}(e)\right)^{p}\right)\right]^{1 / p} \geq \bar{d}
$$

with equality only if $(G, w t)$ is regular.

For the proof, apply the theorem with $\varphi(x)=x^{p}$.

It is intriguing that the edge weights in the corollaries above are allowed to be nonpositive, as long as the degrees come out positive. It is worth noting that $k$ can be 1 in these corollaries; in that case, each edge weight is a degree, and the inequalities turn out to be rather familiar, to inequality aficionados. The inequality in Corollary 2.4, for instance, when $k=1$, is a familiar consequence of Hölder's inequality.

Corollaries 2.3 and 2.4 are only the most obvious applications of Theorem 2.2. It is worth noting that the two are related: as $p$ tends to zero from above, the left-hand side of the inequality in Corollary 2.4 tends to the left-hand side of the inequality in Corollary 2.3. Further, if $w t(e) \geq 0$ for all $e \in E$, the left-hand side of the inequality in Corollary 2.4 nonincreases to the left-hand side in Corollary 2.3, as $p \downarrow 0$, so the latter inequality is "better" than the former, in that case.

The following is a generalization of Corollary 2.3. We have stated Corollary 2.3 separately for esthetic reasons.

Corollary 2.5. Suppose that $\varphi$ takes positive values on a real interval $I$, and $f$ defined by $f(x)=x \log \varphi(x)$ is convex on $I$. For each integer $k \geq 1$ and each $k$-uniform hypergraph $G=(V, E)$, and each weighting wt of the edges of $G$ such that $w t(E)>0$, and the weighted degrees of the vertices are all in $I$,

$$
\left(\Pi_{e \in E}\left(\Pi_{i=1}^{k} \varphi\left(d\left(u_{i}(e)\right)\right)\right)^{w t(e) / k}\right)^{1 / w t(E)} \geq \varphi(\bar{d})
$$

Further, if $f$ is strictly convex on $I$, then equality holds only if $(G, w t)$ is regular.

For the proof, apply Theorem 2.2 with $\varphi$ replaced by $\log \varphi$.

Corollary 2.6. Suppose that $k \geq 1, G$ is a $k$-uniform hypergraph, $\varphi$ and $w t$ satisfy the hypotheses of Corollary 2.5, and wt $(e) \geq 0$ for each $e \in E$. Let $F\left(x_{1}, \ldots, x_{k}\right)=\left(\Pi_{i=1}^{k} \varphi\left(x_{i}\right)\right)^{1 / k}$. Then $*(G, w t, F)$ holds, and if the function $f$ in Corollary 2.5 is strictly convex on $I$, then equality in $*(G, w t, F)$ holds if and only if $(G, w t)$ is regular.

Proof. It is a well-known improvement of the arithmetic mean-geometric mean inequality that if $\lambda_{1}, \ldots, \lambda_{m} \geq 0, \sum_{i=1}^{m} \lambda_{i}=1$, and $a_{1}, \ldots, a_{m} \geq 0$, then

$$
\sum_{i=1}^{m} \lambda_{i} a_{i} \geq \prod_{i=1}^{m} a_{i}^{\lambda_{i}}
$$


Applying this with the numbers $w t(e) / w t(E), e \in E$, playing the roles of the $\lambda_{i}$, and the numbers $\left(\Pi_{j=1}^{k} \varphi\left(d\left(u_{j}(e)\right)\right)\right)^{1 / k}$ playing the roles of $a_{i}$, we have

$$
\frac{1}{w t(E)} \sum_{e \in E} w t(e)\left(\Pi_{j=1}^{k} \varphi\left(d\left(u_{j}(e)\right)\right)\right)^{1 / k} \geq\left(\Pi_{e \in E}\left(\Pi_{j=1}^{k} \varphi\left(d\left(u_{j}(e)\right)\right)\right)^{w t(e) / k}\right)^{1 / w t(E)} .
$$

Noting that $F(\bar{d}, \ldots, \bar{d})=\left(\varphi(\bar{d})^{k}\right)^{1 / k}=\varphi(\bar{d})$, the result now follows from Corollary 2.5.

With a few loose ends hanging, Theorem 2.2 pretty well characterizes those $\varphi$ such that $*(G, w t, F)$ holds, for all $k$-uniform hypergraphs $G$, for admissible $w t$ (see the hypothesis of Theorem 2.2), and for $F$ defined by $F\left(x_{1}, \ldots, x_{k}\right)=\sum_{i=1}^{k} \varphi\left(x_{i}\right)$. Theorem 2.7 makes a start on the same job, for $F$ of the form $F\left(x_{1}, \ldots, x_{k}\right)=\prod_{i=1}^{k} \varphi\left(x_{i}\right)$.

Theorem 2.7. Let $k \geq 1$, let $G=(V, E)$ be a $k$-uniform hypergraph, and let $\varphi$ and $w t$ satisfy the hypotheses of Corollary 2.5. Suppose that $F$ is defined by $F\left(x_{1}, \ldots, x_{k}\right)=\prod_{i=1}^{k} \varphi\left(x_{i}\right)$. Then $*(G, w t, F)$ holds, and if $f(x)=x \log \varphi(x)$ is strictly convex on $I$, then equality holds if and only if $(G, w t)$ is regular.

Proof. Since $g(x)=x^{k}$ is convex and increasing on $[0, \infty)$,

$$
\begin{aligned}
\frac{1}{w t(E)} & \sum_{e \in E} w t(e) \Pi_{i=1}^{k} \varphi\left(d\left(u_{i}(e)\right)\right) \\
& =\frac{1}{w t(E)} \sum_{e \in E} w t(e)\left(\left(\Pi_{i=1}^{k} \varphi\left(d\left(u_{i}(e)\right)\right)\right)^{1 / k}\right)^{k} \\
& \geq\left(\frac{1}{w t(E)} \sum_{e \in E} w t(e)\left(\Pi_{i=1}^{k} \varphi\left(d\left(u_{i}(e)\right)\right)\right)^{1 / k}\right)^{k} \geq \varphi(\bar{d})^{k}=F(\bar{d}, \ldots, \bar{d}),
\end{aligned}
$$

by Corollary 2.6. The condition for equality also follows from Corollary 2.6.

Notice that $\varphi(x)=x$ satisfies the hypothesis of Theorems 2.2 and 2.7, with $I=(0, \infty)$ in the case of Theorem 2.7. The inequalities from Theorem 2.7 with this $\varphi$ seem especially powerful, even though they are not, technically speaking, as "good" as Corollary 2.3. The reader is encouraged to try out a few examples, especially in the case $k=2$. For instance, the inequality from Theorem 2.7 with $\varphi$ being the identity function, with the edge weighting of $C_{4}$ in the proof of Theorem 2.2, is equivalent to the venerable inequality $x^{2}+y^{2} \geq 2 x y$.

\section{Two problems}

(1) Which other simple graphs can play the role that $C_{4}$ plays in Theorem 2.2? Of course, we need not confine the question to simple graphs, but it seems a more attractive problem if we mercifully limit the question, at this point.

By remarks at the end of Section 1, if $0 \in I$ and if $G$, a simple graph, has the property that substituting $G$ for $C_{4}$ in Theorem 2.2 results in a true statement, then any graph of 
which $G$ is a spanning subgraph will have that property. So solving this problem (taking, say, $I=[0, \infty)$ ) will amount to finding the minimal graphs of each order, if any, that have that property. We wonder if all cycles have that property, and if any trees do.

(2) For $1 \leq s \leq k$, let $A(k, s)$ denote the collection of $s$-subsets of $\{1, \ldots, k\}$ and let $P_{k, s}$ be defined by $P_{k, s}\left(x_{1}, \ldots, x_{k}\right)=\sum_{B \in A(k, s)} \Pi_{i \in B} x_{i} ; P_{k, s}$ is the "standard" symmetric polynomial of degree $s$ of $k$ variables. Theorems 2.2 and 2.7 are about $*(G, w t, F)$ being satisfied for all $k$-uniform $G$ and all admissible $w t$, with $F$ of the form $F\left(x_{1}, \ldots, x_{k}\right)=$ $P_{k, s}\left(\varphi\left(x_{1}\right), \ldots, \varphi\left(x_{k}\right)\right)$, for $s=1$ and $s=k$. The game we are playing is to ask for which $\varphi$ is $*(G, w t, F)$ true for all $(G, w t)$. Attacking this question for $1<s<k$ looks quite like a job. Here is a smaller, more approachable question: so far, for $s=1$ and $s=k$, if $I=[0, \infty)$, the functions $\varphi(x)=x^{p}, p>0$, have turned out to be "good;" will these functions be "good" for all $s$ and $k$ ?

\section{References}

[1] G. H. Hardy, J. E. Littlewood, and G. Pólya, Inequalities, Cambridge University Press, Cambridge, 1934.

[2] P. D. Johnson Jr. and R. L. Perry, Inequalities relating degrees of adjacent vertices to the average degree, European J. Combin. 7 (1986), no. 3, 237-241.

P. D. Johnson Jr.: Department of Mathematics and Statistics, College of Science and Mathematics, Auburn University, AL 36849-5307, USA

E-mail address: johnspd@auburn.edu

R. N. Mohapatra: Department of Mathematics, College of Arts and Sciences, University of Central Florida, Orlando, FL 32816-1364, USA

E-mail address: ramm@pegasus.cc.ucf.edu 


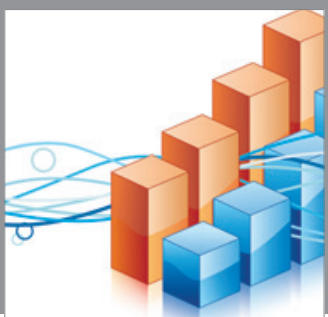

Advances in

Operations Research

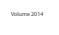

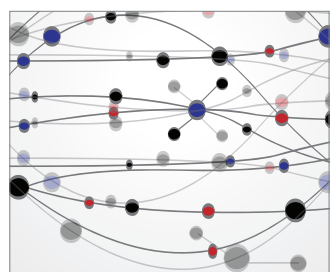

\section{The Scientific} World Journal
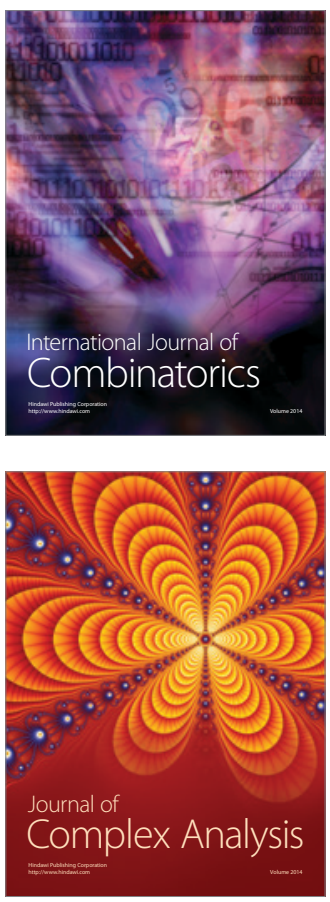

International Journal of

Mathematics and

Mathematical

Sciences
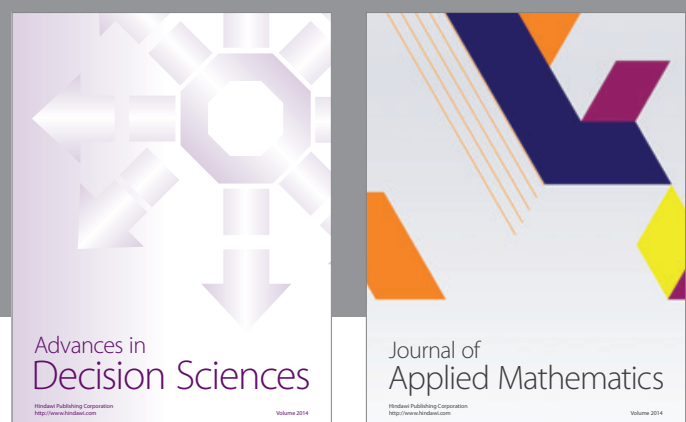

Journal of

Applied Mathematics
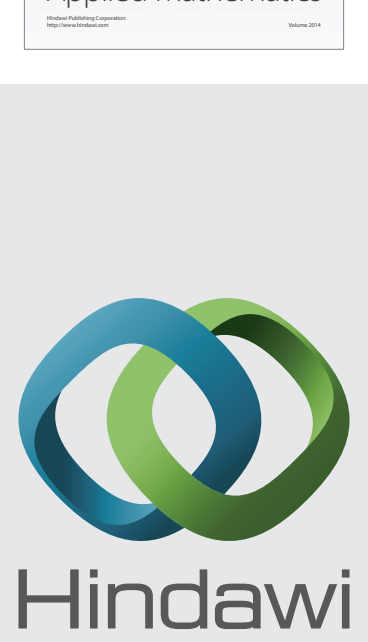

Submit your manuscripts at http://www.hindawi.com
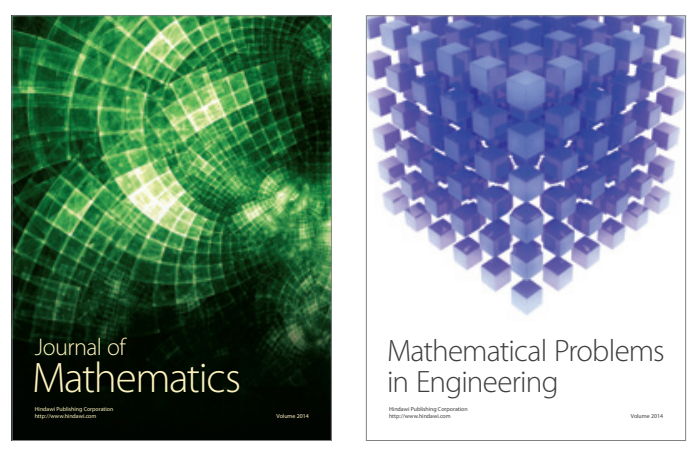

Mathematical Problems in Engineering
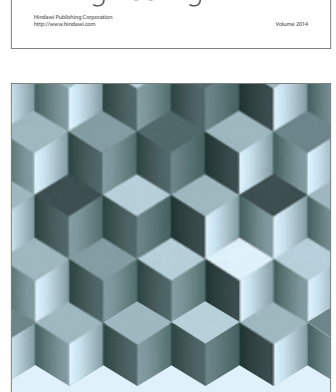

Journal of

Function Spaces
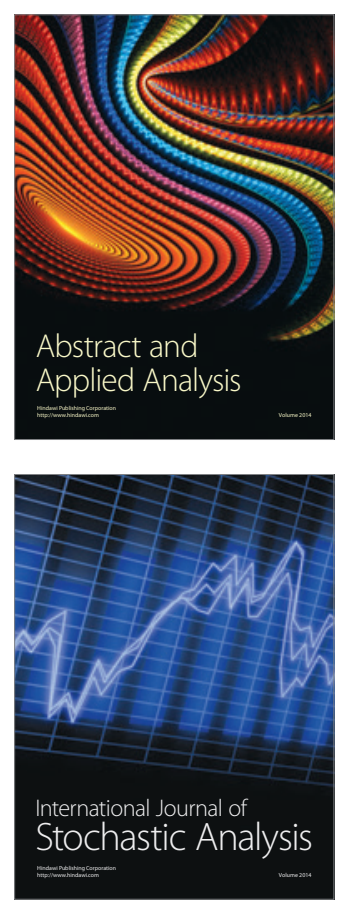

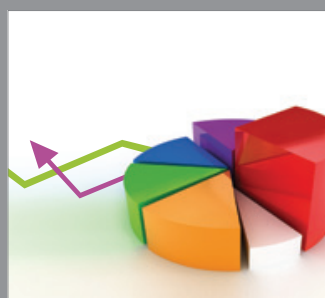

ournal of

Probability and Statistics

Promensencen
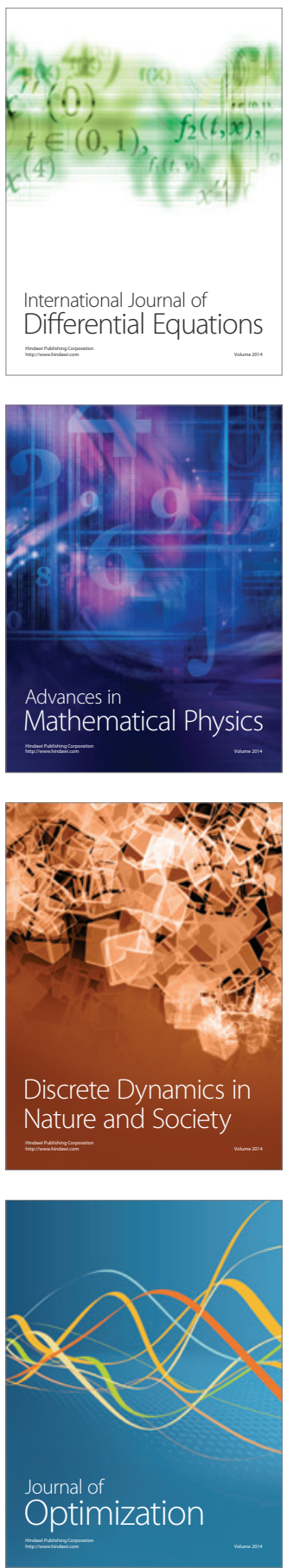